Linguistic Issues in Language Technology - LiLT Submitted, January 2012

\title{
Parallel Syntactic Annotation in CReST
}

Sandra Kübler

Eric Baucom

Matthias Scheutz 



\title{
Parallel Syntactic Annotation in CReST
}

\author{
SANDra KÜBler, Indiana University ERIC BAucom, Indiana \\ University Matthias Scheutz, Tufts University
}

\begin{abstract}
In this paper, we introduce the syntactic annotation of the CReST corpus, a corpus of natural language dialogues obtained from humans performing a cooperative, remote search task. The corpus contains the speech signals as well as transcriptions of the dialogues, which are additionally annotated for dialogue structure, disfluencies, and for syntax. The syntactic annotation comprises POS annotation, Penn Treebank style constituent annotations, dependency annotations, and combinatory categorial grammar annotations. The corpus is the first of its kind, providing parallel syntactic annotation based on three different grammar formalisms for a dialogue corpus. All three annotations are manually corrected, thus providing a high quality resource for linguistic comparisons, but also for parser evaluation across frameworks.
\end{abstract}

LiLT Volume 7, Issue 4, January 2012.

Parallel Syntactic Annotation in CReST.

Copyright (C) 2012, CSLI Publications. 


\section{Introduction}

Despite the increasing interest in spoken natural language interactions in dialogue systems and with robots and other types of artificial agents, there is a surprising lack of corpora that contain typical natural language dialogue interactions in naturalistic environments. Yet, such corpora would be of great utility for developing robust components for natural processing systems for artificial agents. Specifically, they could be used to train speech recognizers and parsers, develop methods for coping with common disfluencies as they frequently occur in spontaneous speech, and define appropriate semantic formalisms that capture different non-truth-functional aspects of typical utterances. Moreover, they could be used as benchmarks for the systematic comparison of different speech recognizers, parsers, and semantic analyzers.

In this paper, we introduce such a corpus - the Indiana "Cooperative Remote Search Task" (CReST) corpus - which was specifically developed to fill this void. Different from standard corpora such as the Wall Street Journal corpus of the Penn Treebank Marcus et al. (1993), CReST was developed with different parallel syntactic annotations in mind to specifically facilitate linguistic comparisons across grammar formalisms as well as comparison of different types of parsers (among others). As such, the corpus includes three different syntactic annotations: constituent, dependency, and combinatory categorial grammar (CCG). We start by briefly describing the corpus, followed by a description of the three types of annotation. Then we also give some natural language examples that set the CReST corpus apart from other existing corpora and point to the utility for studying and evaluation of natural language processing components in the context of naturalistic spoken language exchanges.

\section{The CReST Corpus}

The Indiana "Cooperative Remote Search Task" (CReST) corpus (Eberhard et al., 2010) is a corpus of natural language dialogues obtained from humans performing a cooperative, remote search task in which one person outside the search environment (director) directed a person inside the environment (searcher). The director guided the searcher through the search environment, for which the director had a map, in order to find different colored boxes, enter them on the map, and place blocks in them. The director was fitted with a free-head eyetracker, and s/he was recorded by a microphone positioned between the director and the telephone's speaker. The searcher wore a helmet with a cordless phone and a light-weight digital video camera that 
recorded their movement through the environment as viewed from the searcher's perspective and provided a second audio recording of the spoken dialogue.

CReST is similar in the task setting to the HCRC Map Task Corpus (Thompson et al., 1996). However, in CReST, the dyads do not share visual information. Thus they have to communicate information verbally in both directions to perform the tasks.

The multi-modal corpus consists of 23 dialogues. The text highlights the differences between formal written and naturally occurring language, as it is rife with directives, disfluencies, corrections, ungrammatical sentences, wrong-word substitutions, and various other constructions that are missing from written text corpora. In total, there are 40083 words in 5872 sentences.

The corpus contains the speech signals as well as transcriptions of the dialogues, which are additionally annotated for dialogue structure, disfluencies, and for syntax. The syntactic annotation comprises POS annotation, Penn Treebank (Marcus et al., 1993) style constituent annotations, dependency annotations based on the dependencies of pennconverter (Johansson and Nugues, 2007), as well as combinatory categorial grammar annotations based on the algorithm provided by Hockenmaier and Steedman (Hockenmaier and Steedman, 2007).

\subsection{Annotation}

On the dialogue level, the corpus was annotated for dialogue structure and for disfluencies. Utterances were divided into separate dialogue moves, based on the classification developed by Carletta et al. (1997) for coding task-oriented dialogues. Their scheme views utterances as moves in a conversational game and classifies utterances into three basic move categories: Initiation, Response, and Ready. Initiation is further divided into INSTRUCT, EXPLAIN, QUERY-YN, QUERY-W, CHECK, and ALIGN. The category Response includes ACKNOWLEDGE, replies to whquestions REPLY-WH, and yes or no replies REPLY-Y, REPLY-N.

The POS annotation is based on the Penn Treebank POS tagset (Santorini, 1990), with a small number of new POS tags added to describe typical characteristics of spoken language:

- AP for adverbs that serve for answering questions, such as yes, no, or right.

- DDT for substituting demonstratives, such as in that is correct.

- VBI for imperatives, such as turn left.

- XY for non-words or interrupted words.

The first sentence in Figure 1 shows an example of a sentence with 
three new POS tags. Another modification of the tagset concerns informal contractions such as in you 're gonna wanna turn to the right?, which are kept as single words. As a consequence, they are assigned combinations of tags, such as VBG+TO. The second sentence below shows an example of such a contraction.

$\begin{array}{llll}\text { yeah } & \text { AP } & \text { you } & \text { PRP } \\ \text { let } & \text { VBI } & \text { 're } & \text { VBP } \\ \text { 's } & \text { PRP } & \text { gonna } & \text { VBG }+ \text { TO } \\ \text { do } & \text { VB } & \text { find } & \text { VB } \\ \text { that } & \text { DDT } & \text { a } & \text { DT } \\ \text { yeah } & \text { UH } & \text { pink } & \text { JJ } \\ & & \text { box } & \text { NN }\end{array}$

FIGURE 1 Two example sentences with POS annotation

\section{Syntactic Annotation}

In addition to the levels of annotation described above, the corpus is annotated in parallel for constituent, dependency, and combinatory categorial grammar (CCG). The annotations are based on automatic annotations, either by a parser, or by conversion, and consequently manually checked. This provides a unique resource for English syntactic annotation, which allows the comparison of the different syntactic annotations for the same sentence as well as the comparison of parsers trained on the different syntactic annotations. Since we used the constituent version as the underlying basis for the other two formats, we conjecture that we reach maximal consistency between the different annotation schemes. The treebank is similar to the Turin University Treebank for Italian (Bos et al., 2009, Bosco and Lombardo, 2004), which covers annotations based on the same grammar formalisms, but is more restricted in size.

\subsection{Constituent Annotation}

The constituent annotation is based on the Penn Treebank annotations (Santorini, 1991). The annotation concentrates on the surface form. For this reason, we did not annotate empty categories and traces. Since the collaborative task involved maneuvering in an unknown environment, the annotation of grammatical functions concentrates on the functions subject (SBJ), predicate (PRED), locative (LOC), direction (DIR), and temporal (TMP).

Modifications of the annotation scheme were necessitated by the 
spontaneous speech data: For many sentences, the high frequency of disfluencies prevented a complete grammatical analysis. In such cases, the maximal possible grammatical string was annotated. The ungrammatical elements were annotated as fragments (FRAG) on the lowest level covering all the disfluencies and then integrated into the tree structure.

\subsection{Dependency Annotation}

The dependency annotation is based on the automatic dependency conversion from Penn-style constituents by pennconverter (Johansson and Nugues, 2007). This means that we used the same style of annotation, but not the converter. Instead, the sentences were parsed by a dependency parser trained on the Penn dependencies; then they were corrected manually. We made small changes to the annotation scheme: For coordinations, we decided to attach both the conjunction and the second conjunct to the first conjunct. The reason for this decision lies in an attempt to reach consistency with coordinations without conjunctions, for which the second conjunct would have to be dependent on the first conjunct. This treatment differs, for example, from the dependency theory by Mel'čuk (2003), who describes the conjunction as dependent of the first conjunct. In Mel'čuk's analysis, the second conjunct is dependent on the conjunction if available or on the first conjunct when there is no conjunction. However, Mel'cuk (1988) lists cases where the presence of the second conjunct in a subject enforces plural agreement, which would argue for accepting the second conjunct as the head. Hudson (1990), in contrast, argues for a phrase-based, parallel treatment of conjuncts outside the dependencies. Since McDonald and Nivre (2007) show that different parsers prefer different decisions concerning the head of a coordination, there is no computational reason for preferring one analysis over another. We also decided to make subordinating conjunctions dependent on the finite verb of the subordinate clause, which in turn is dependent on the verb of the matrix clause.

\subsection{Combinatory Categorial Grammar Annotation}

To obtain our CCG annotations, we automatically converted the Pennstyle constituent annotations following the conversion by Hockenmaier and Steedman (2007) for the Penn Treebank. We then manually correct the annotations. To determine the constituent types for the conversion, heuristics are required. Hockenmaier and Steedman adapted theirs from the head-finding rules developed by Collins (1999) and Magerman (1994). Ungrammatical sentences are processed automatically once their constituent types are determined from the heuristics, 
although in such cases the terms "head," "complement," and "adjunct" lose some of their meaning.

Since CReST uses additional POS tags, we added these as head candidates for FRAG and VP nodes. The heuristics used to distinguish complements and adjuncts rely on the presence of grammatical function categories, many of which are not coded in CReST. We had to disambiguate those manually. Following Hockenmaier and Steedman, we allow forward and backward rule application, and restrict the combinatory rules for CCG to forward and backward composition and backward crossing composition. This restriction sometimes leads to a proliferation in categories, especially given the fluid nature of syntax for dialogues. In our estimation, this is preferable to allowing more combinatory rules, even though it may lead to unexpected pairings of word and category in some (typically ungrammatical) cases.

\section{Selected Phenomena in CReST}

In this section, we present examples for phenomena that distinguish the textual basis of the CReST corpus from the Penn Treebank. Thus, we focus on phenomena typical for spontaneous speech that do not occur in the Penn Treebank or are less frequent there. For the sentences, we present the syntactic analyses in all three syntactic formalisms.

\subsection{Questions and Imperatives}

While the Penn Treebank consists mostly of statements, CReST has a significant number of questions and imperatives: Among the 5872 sentences, there are 843 questions and 550 imperatives. In comparison to QuestionBank (Judge et al., 2006), CReST has a high number of yes/no questions. The constituent annotation for a typical question is shown in Figure 2, the corresponding dependency annotation in Figure 3 and the CCG annotation in Figure 4.

Since CReST is based on cooperative dialogues, many questions are backchannels rather than requests for information. Such questions often have the non-inverted word order of a statement in combination with raising intonation. In the constituent annotation, they are projected to an $\mathrm{S}$ node, but they end in a question mark. We show an example in Figure 5 .

An example of an imperative is shown in Figures 6, 7, and 8.

\subsection{Fragments and Corrections}

CReST sentences also have a high percentage of fragmented utterances and corrections, which are typical for spontaneous speech. In the constituent annotation, fragments are grouped under a FRAG node and 


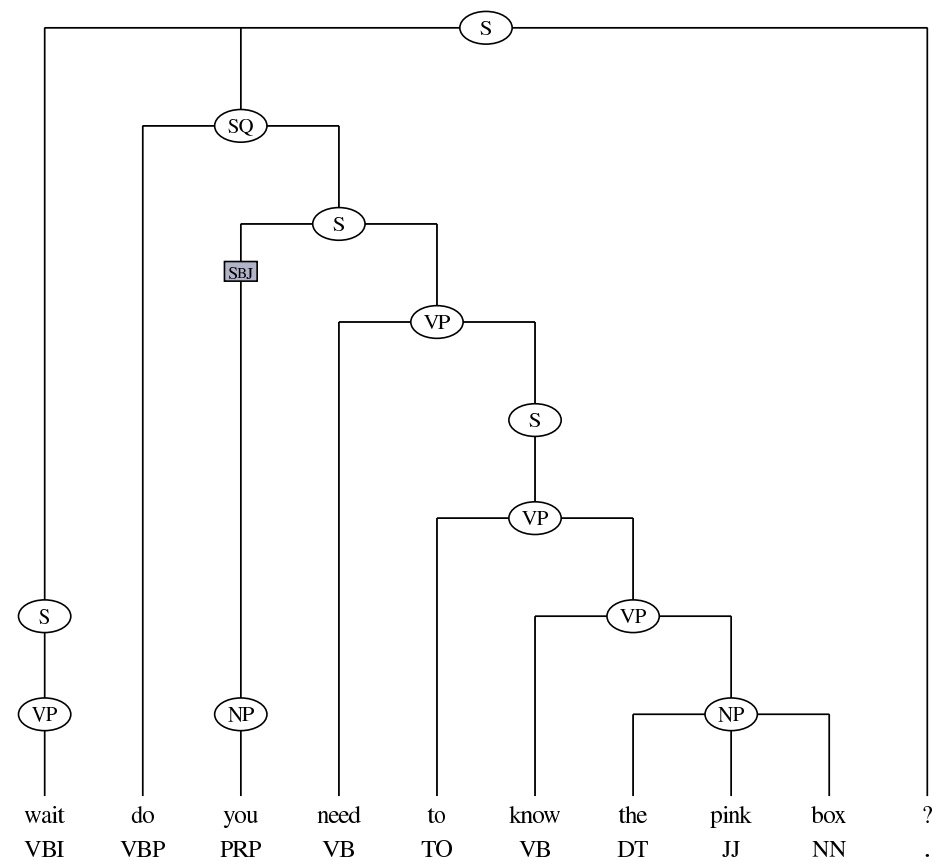

FIGURE 2 An example of the constituent annotation of a question

integrated into the remainder of the sentence. The only exceptions are non-words, which receive the POS tag XY; those are attached directly to the surrounding constituent. In the dependency annotation, fragments remain unattached, and ungrammatical dependencies are starred. Fragments are treated as adjuncts in the CCG annotation, allowing them to seek and then return the head node by virtue of their function status. Figures 9, 10, and 11 show a sentence containing fragments, and Figures 12, 13, and the first example in Figure 14 show a sentence containing a correction.

\subsection{Extraposition and Coordination}

Spontaneous language often shows overt editing or a high compression of information in elliptical constructions. Such phenomena are generally not present in written language, where there is less need for speed and conciseness. Below, we show an example in which a short answer to the left, is then elaborated further. In such cases, we decided to treat the 


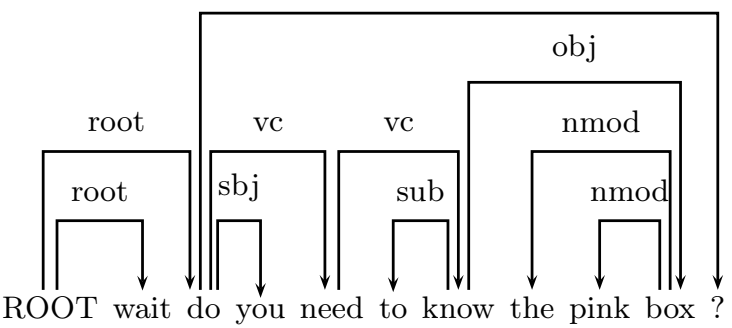

FIGURE 3 An example of the dependency annotation of a question

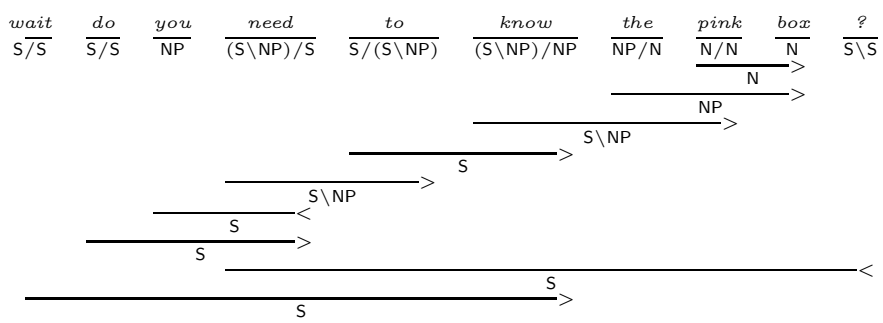

FIGURE 4 An example of the CCG annotation of a question

first answer as a fronted element of the following clause. Figure 15 shows the constituent annotation of a sentence with a fronted prepositional phrase, and Figure 16 the dependency version. The CCG version is shown as the second example in Figure 14. In the CCG derivation, we see the repeated determiner the in the fragment taking the unusual categories $S / S$ and $S \mid S$. Here, the CCG conversion algorithm identifies the as the head of the fragment FRAG from the original constituent annotation. The fragment itself is an adjunct to the $\mathrm{S}$ node in the constituent annotation, so its CCG category of $S / S$ percolates down. Alternatively, if the had the usual $N P / N$ categories, it would not be consistent with the original constituent annotation, where the sequence of the - the - is treated as a FRAG under the $\mathrm{S}$ node.

The sentence shown in Figures 17 and 18 illustrates an elliptical coordination. The $\mathrm{CCG}$ version is shown as the third example in Figure 14 .

\section{Conclusion}

We presented the CReST corpus developed from natural language dialogue data, which was collected as part of a remote search task between two humans as it naturally occurs in a variety of domains. In addition to the audio data, the corpus contains fully transcribed text 


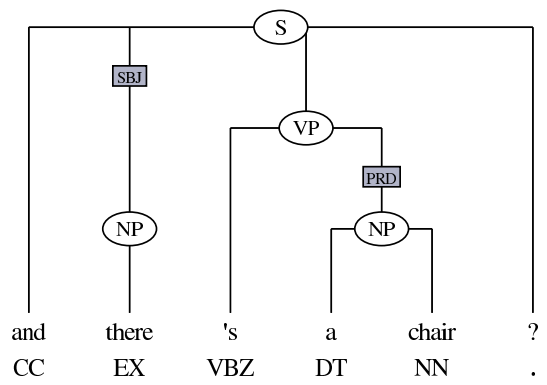

FIGURE 5 An example of the constituent annotation of a backchannel question

with disfluency annotations and, for the purpose of this paper most critically, three different syntactic annotations based on constituent, dependency, and combinatory categorial grammar. The corpus is the first of its kind, providing parallel syntactic annotation based on three different grammar formalisms for a sizeable number of sentences. This parallel annotation allows for the direct comparison and evaluation of linguistic phenomena as well as of parsers based on the three grammar formalisms in an unprecedented way in a naturalistic task. We believe that such comparisons are not only of great utility for the linguistics and computational linguistics community, but also for artificial intelligence and robotics researchers who intend to develop complete natural language understanding systems for agents that are intended to interact with humans in natural ways.

\section{Acknowledgments}

This work is based on research supported by the US Office of Naval Research (ONR) Grant \#N00014-10-1-0140 and the ONR MURI grant \#N00014-07-1-1049.

\section{References}

Bos, Johan, Cristina Bosco, and Alessandro Mazzei. 2009. Converting a dependency treebank to a categorial grammar treebank for Italian. In Proceedings of the Eighth Workshop on Treebanks and Linguistic Theories (TLT). Milan, Italy.

Bosco, Cristina and Vincenzo Lombardo. 2004. Dependency and relational structure in treebank annotation. In Proceedings of the COLING Workshop on Recent Advances in Dependency Grammar, pages 9-16. Geneva, Switzerland. 
Carletta, Jean, Stephen Isard, Amy Isard, Gwyneth Doherty-Sneddon, Jacqueline Kowtko, and Anne Anderson. 1997. The reliability of a dialogue structure coding scheme. Computational Linguistics 23(1):13-31.

Collins, Michael. 1999. Head-Driven Statistical Models for Natural Language Parsing. Ph.D. thesis, University of Pennsylvania.

Eberhard, Kathleen, Hannele Nicholson, Sandra Kübler, Susan Gunderson, and Matthias Scheutz. 2010. The Indiana "Cooperative Remote Search Task" (CReST) Corpus. In Proceedings of the Seventh International Conference on Language Resources and Evaluation (LREC). Valetta, Malta.

Hockenmaier, Julia and Mark Steedman. 2007. CCGbank: A Corpus of CCG derivations and dependency structures extracted from the Penn Treebank. Computational Linguistics 33(3):355-396.

Hudson, Richard. 1990. English Word Grammar. Blackwell.

Johansson, Richard and Pierre Nugues. 2007. Extended constituent-todependency conversion for English. In Proceedings of NODALIDA 2007. Tartu, Estonia.

Judge, John, Aoife Cahill, and Josef van Genabith. 2006. QuestionBank: Creating a corpus of parse-annotated questions. In Proceedings of the 21st International Conference on Computational Linguistics and 44th Annual Meeting of the Association for Computational Linguistics (COLING$A C L)$, pages 497-504. Sydney, Australia.

Magerman, David M. 1994. Natural Language Parsing as Statistical Pattern Recognition. Ph.D. thesis, Stanford University.

Marcus, Mitchell, Beatrice Santorini, and Mary Ann Marcinkiewicz. 1993. Building a large annotated corpus of English: The Penn Treebank. Computational Linguistics 19(2):313-330.

McDonald and Joakim Nivre. 2007. Characterizing the errors of data-driven dependency parsing models. In Proceedings of the 2007 Joint Conference on Empirical Methods in Natural Language Processing and Computational Natural Language Learning (EMNLP-CoNLL), pages 122-131. Prague, Czech Republic.

Mel'čuk, Igor A. 1988. Dependency Syntax: Theory and Practise. SUNY Series in Linguistics. Albany, NY: State University of New York Press.

Mel'čuk, Igor A. 2003. Levels of dependency in linguistic description: Concepts and problems. In V. Ágel, L. M. Eichinger, H. W. Eroms, P. Hellwig, H. J. Heringer, and H. Lobin, eds., Dependency and Valency. An International Handbook of Contemporary Research, vol. 1, pages 188-229. de Gruyter.

Santorini, Beatrice. 1990. Part-of-speech tagging guidelines for the Penn Treebank Project. Department of Computer and Information Science, University of Pennsylvania, 3rd Revision, 2nd Printing.

Santorini, Beatrice. 1991. Bracketing guidelines for the Penn Treebank Project. Department of Computer and Information Science, University of Pennsylvania. 
Thompson, Henry, Anne Anderson, Ellen Gurman Bard, Gwyneth DohertySneddon, Alison Newlands, and Cathy Sotillo. 1996. The HCRC Map Task Corpus: Natural dialogue for speech recognition. In Proceedings of the ARPA Human Language Technology Workshop. Plainsboro, NJ.

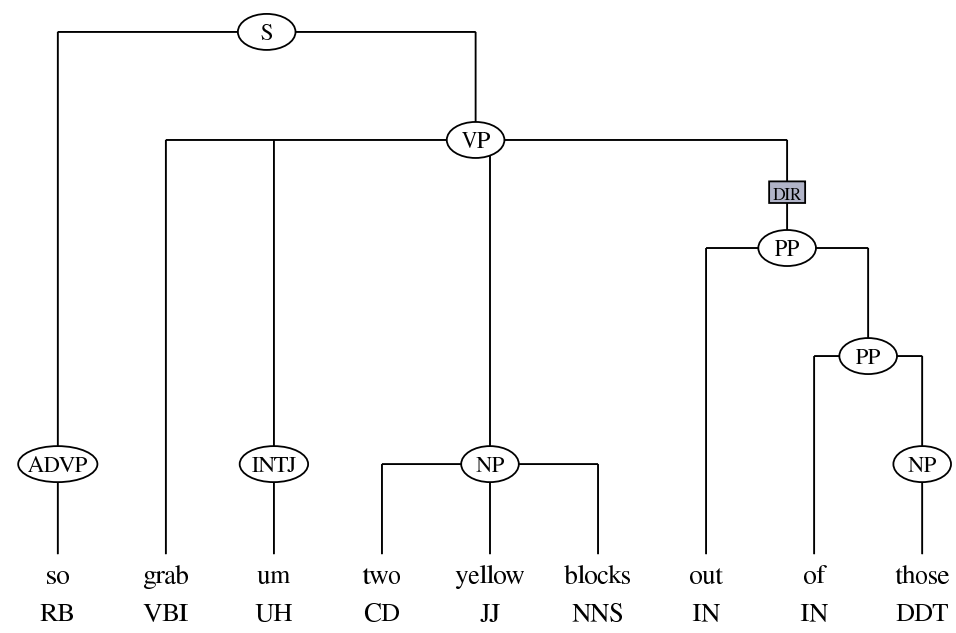

FIGURE 6 An example of the constituent annotation of an imperative

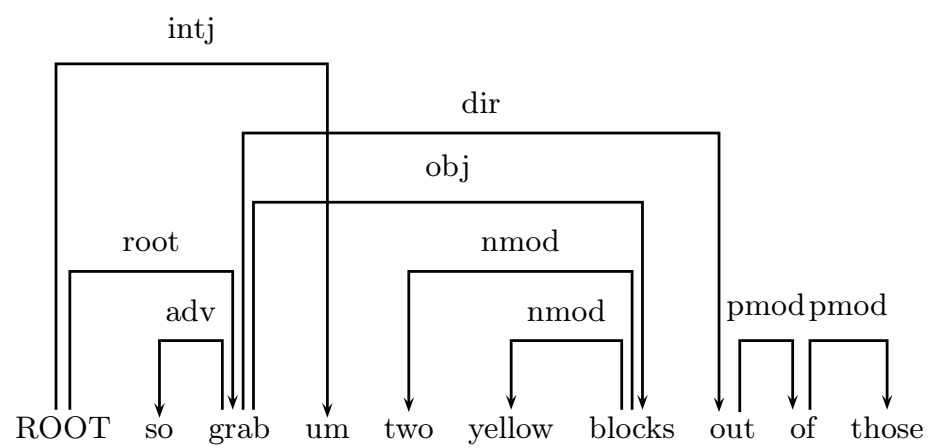

FIGURE 7 An example of the dependency annotation of an imperative 
12 / LiLT volume 7, issue 4

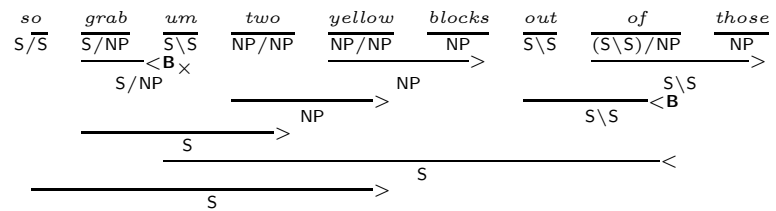

FIGURE 8 An example of the CCG annotation of an imperative

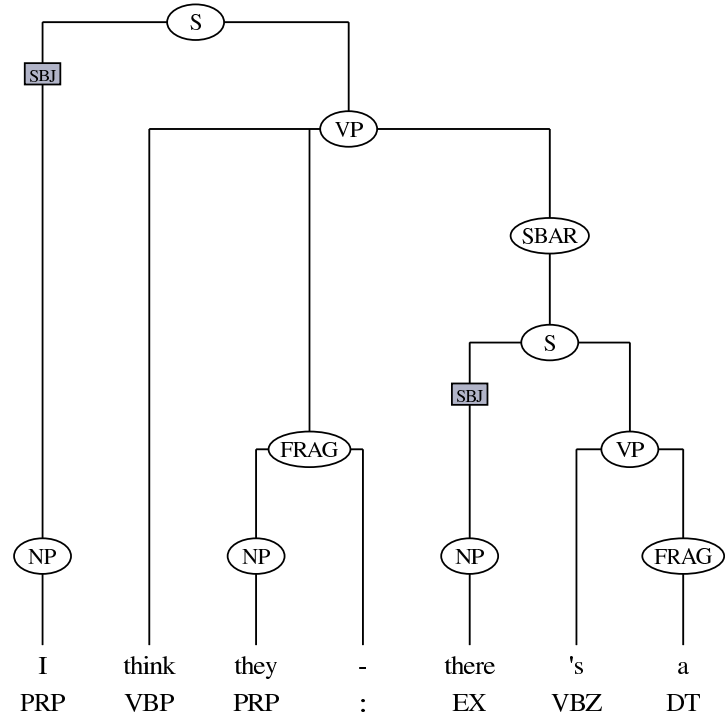

FIGURE 9 An example of the constituent annotation of a fragment

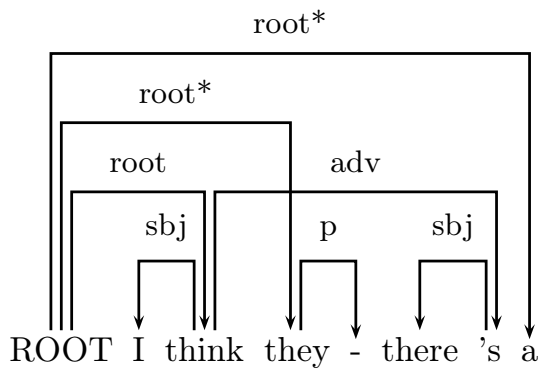

FIGURE 10 An example of the dependency annotation of a fragment 


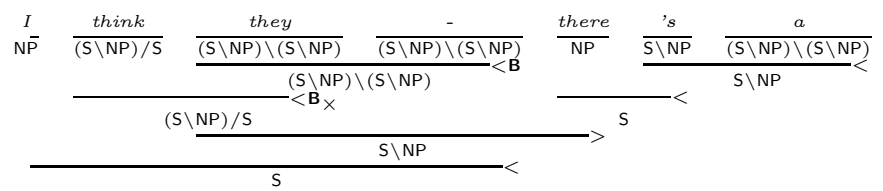

FIGURE 11 An example of the CCG annotation of a fragment

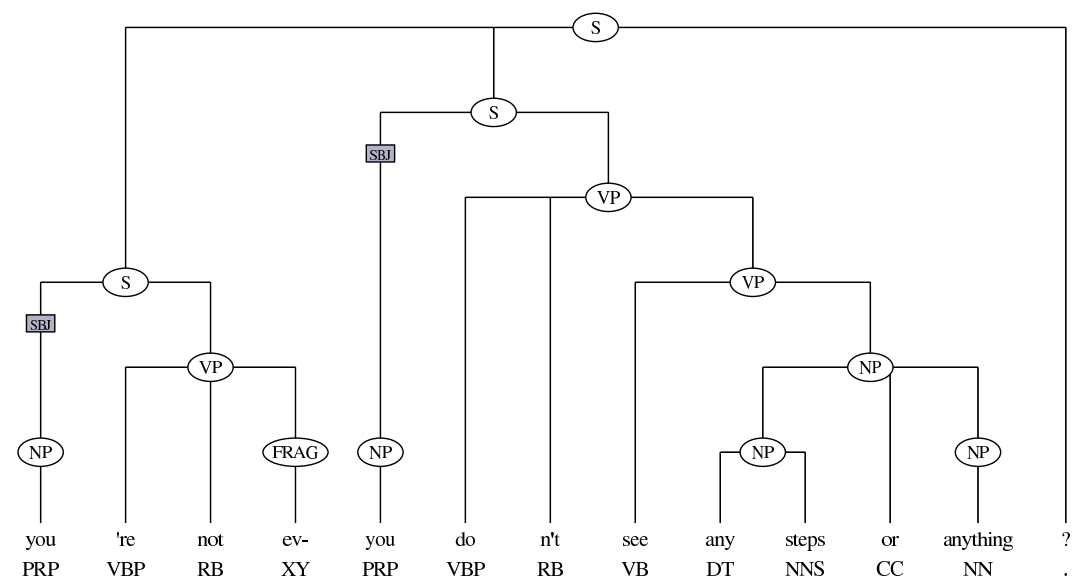

FIGURE 12 An example of the constituent annotation of a correction

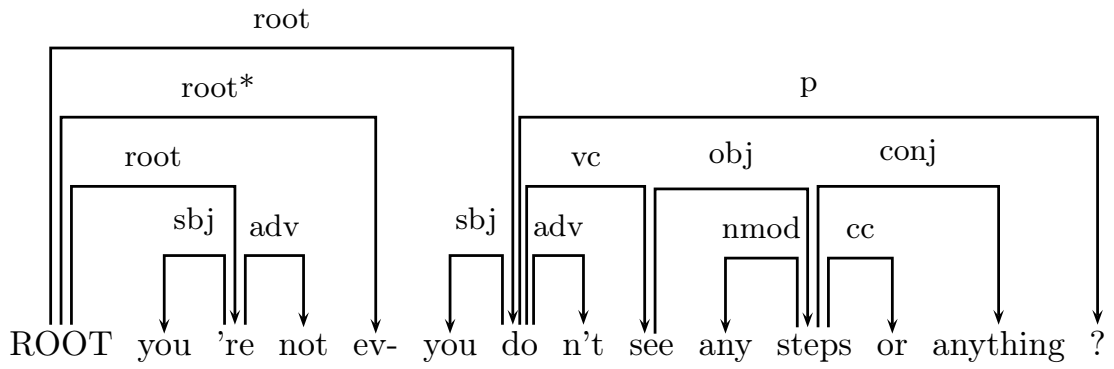

FIGURE 13 An example of the dependency annotation of a correction 


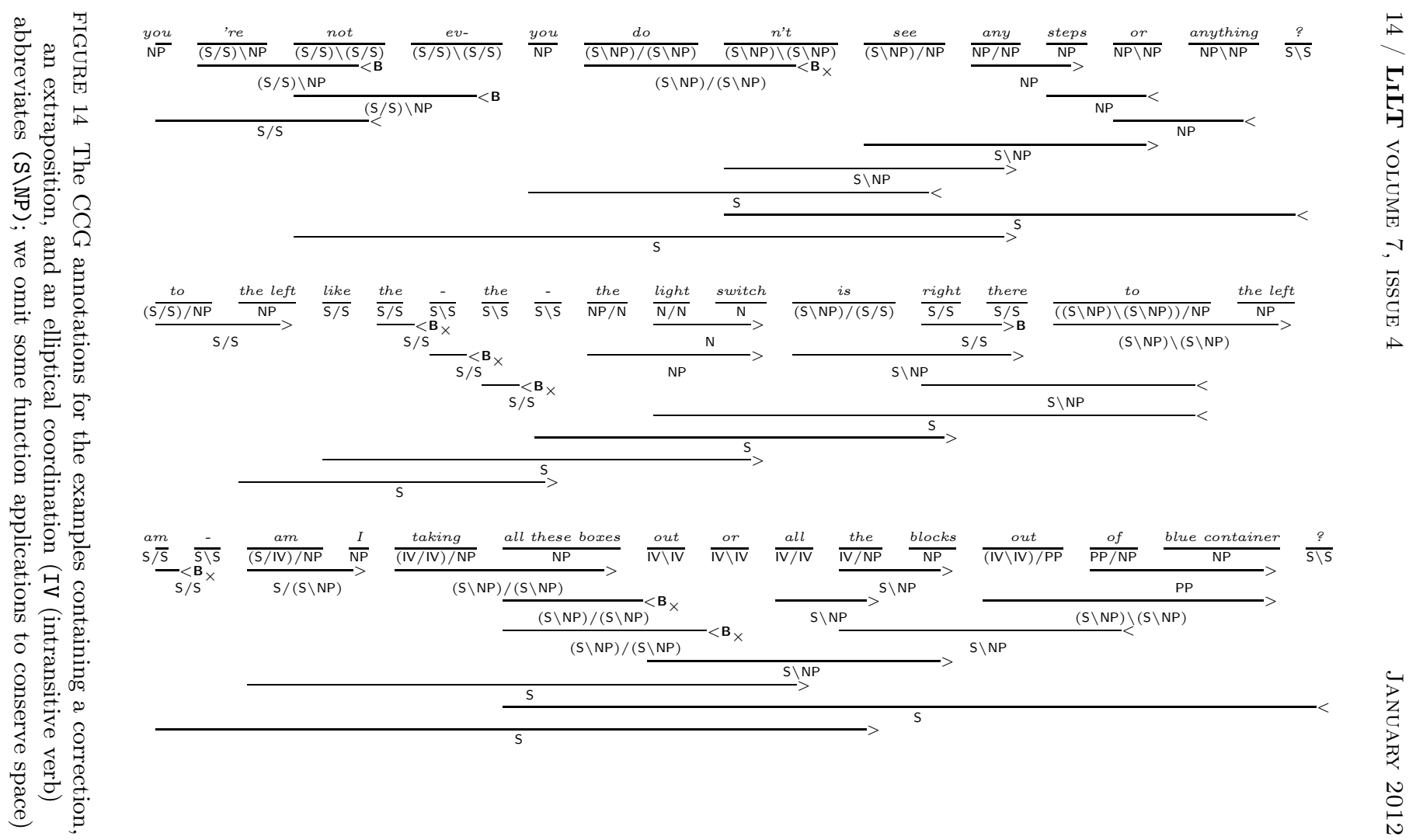




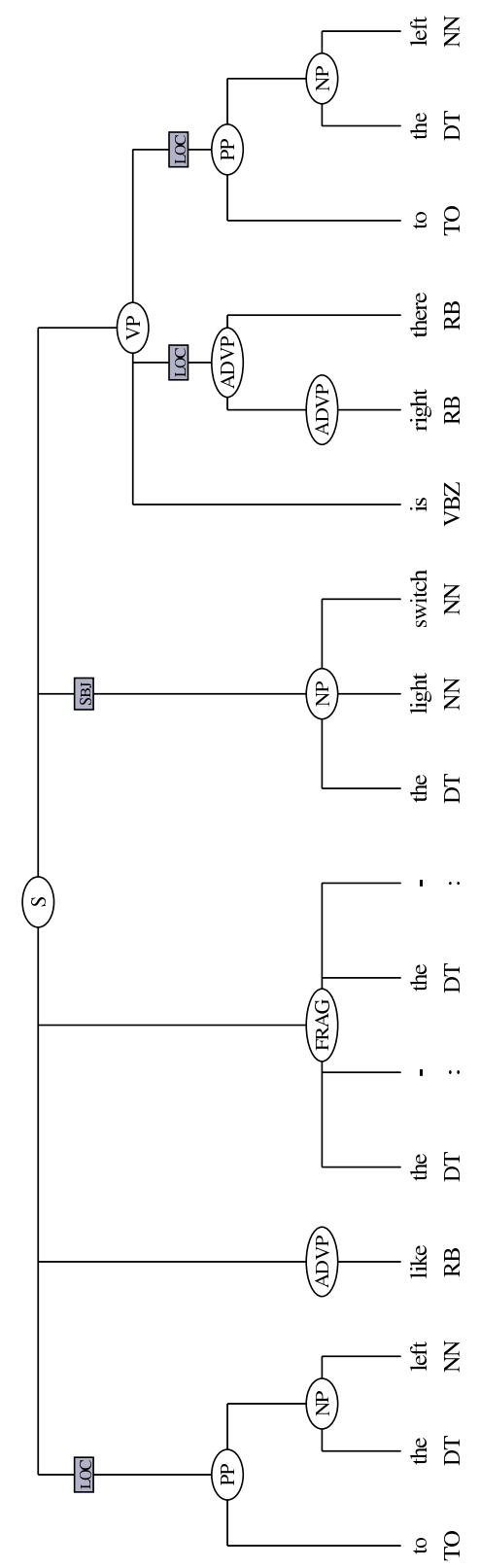

FIGURE 15 An example of the constituent annotation of an extraposition 


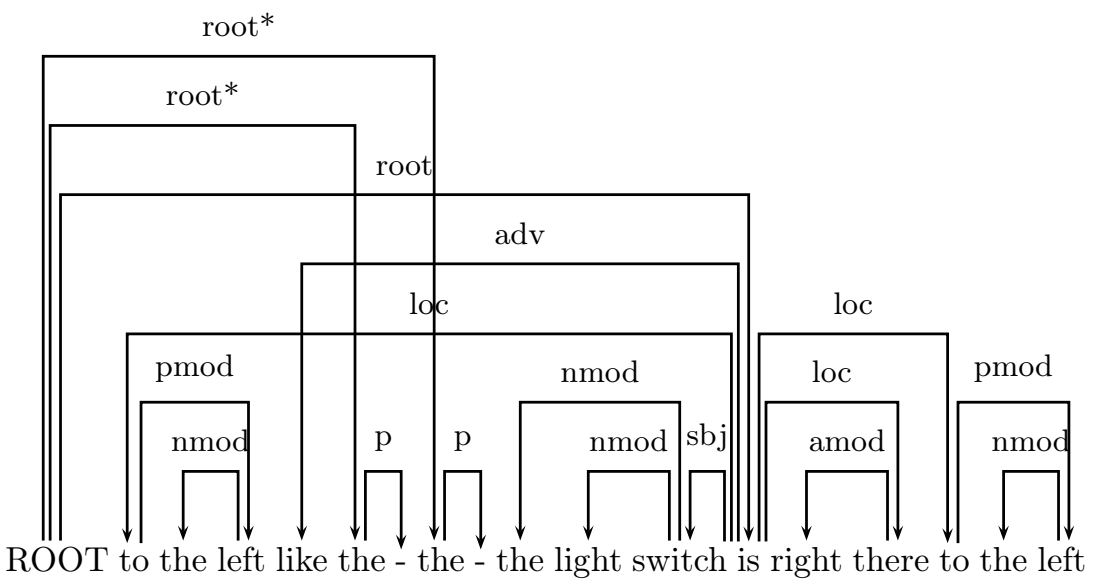

FIGURE 16 An example of the dependency annotation of an extraposition 


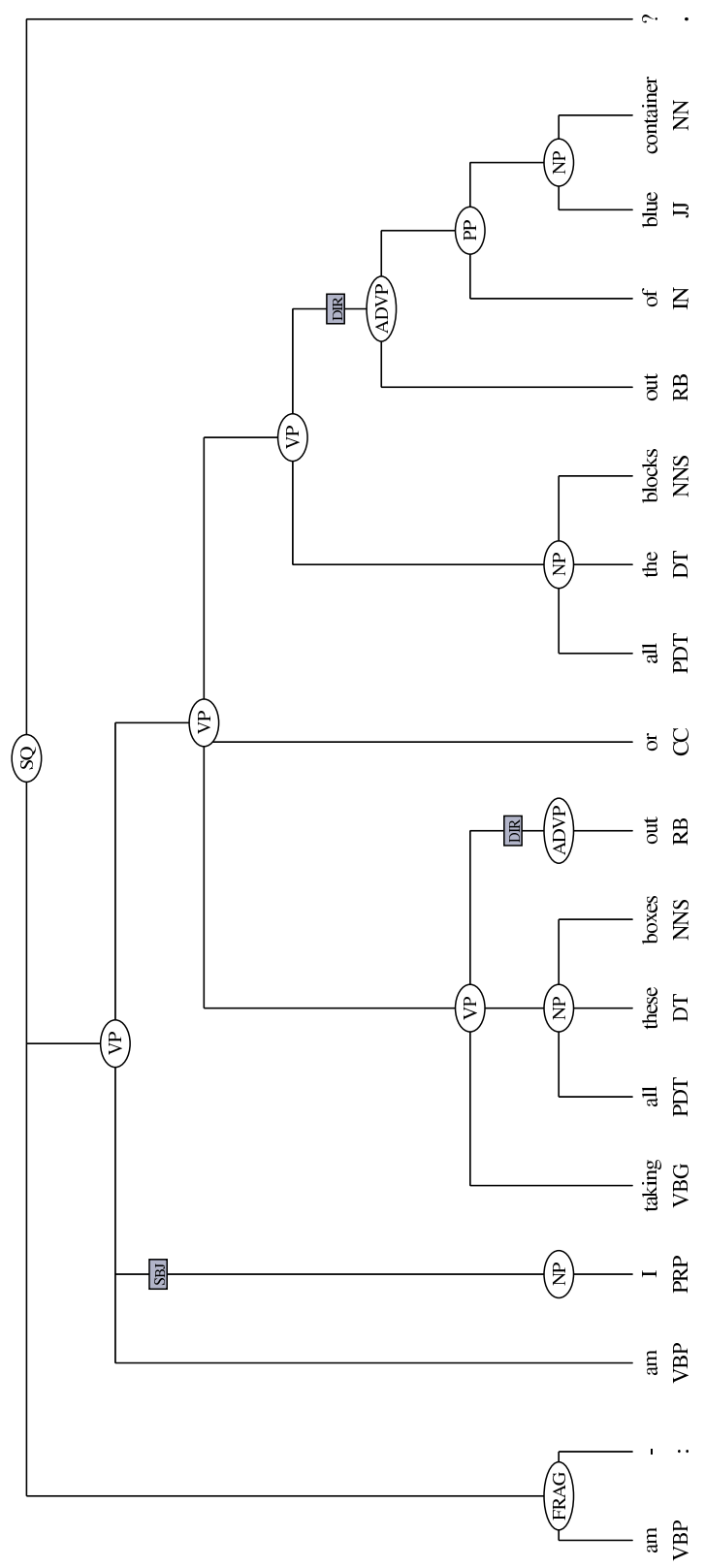

FIGURE 17 An example of the constituent annotation of an elliptical coordination 


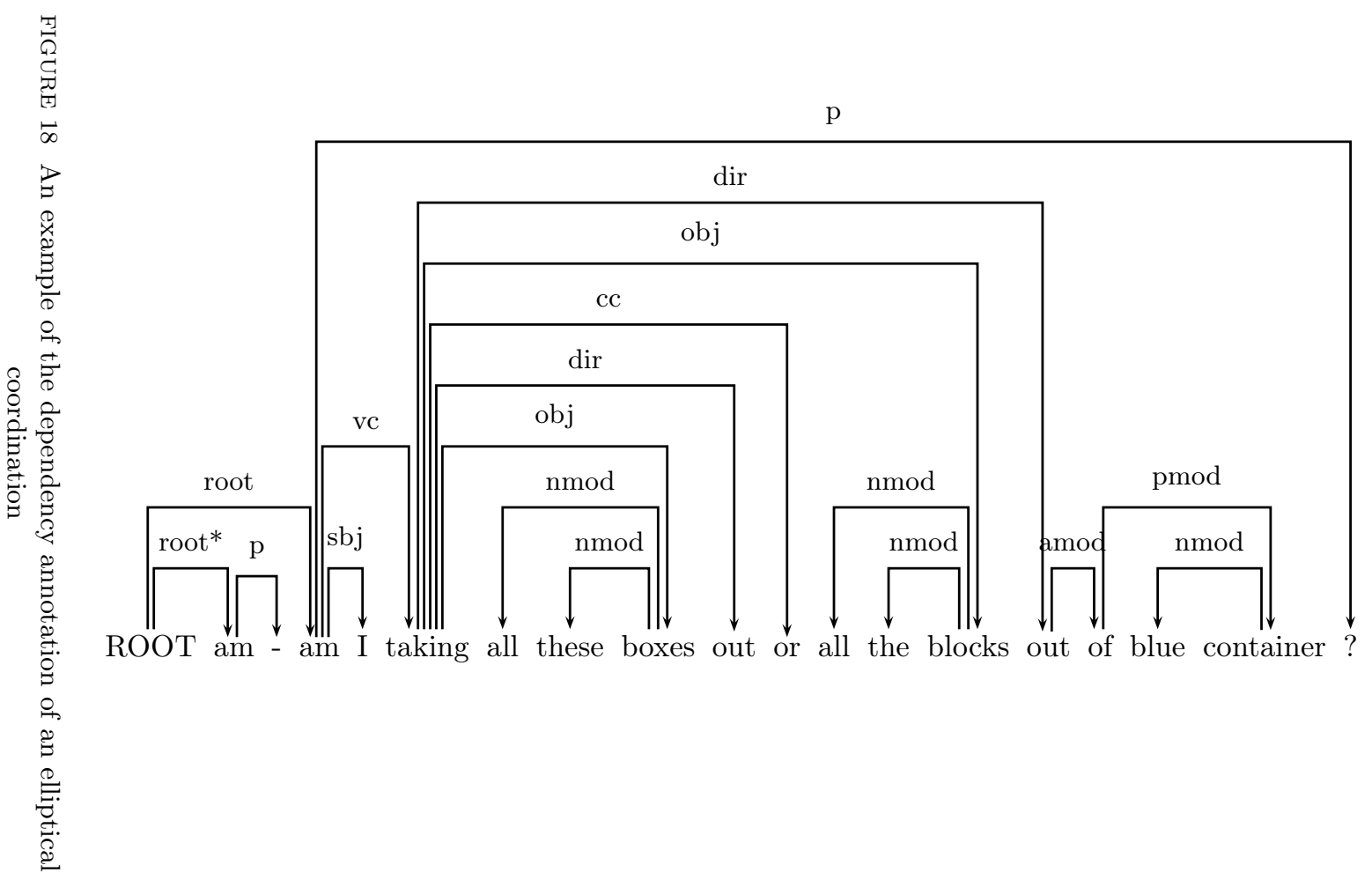

